\title{
Electrocardiographic P-wave Delineation Based on Adaptive Slope Gaussian Detection
}

\author{
Francisco González ${ }^{1}$, Raúl Alcaraz ${ }^{2}$, José J Rieta ${ }^{1}$ \\ ${ }^{1}$ BioMIT.org, Electronic Engineering Department, Universitat Politecnica de Valencia, Spain \\ ${ }^{2}$ Research Group in Electronic, Biomedical and Telecomm. Eng., Univ. of Castilla-La Mancha, Spain
}

\begin{abstract}
The study of the P-wave on the electrocardiogram is essential in the characterization of atrial conduction defects that may anticipate cardiac pathologies, such as atrial fibrillation. This evidence exhibits the need to develop reliable methods for accurate automatic delineation of $P$ waves. Many different strategies for delineating $P$-waves have been introduced. Nonetheless, they all share the same principle of smoothing aggressively the P-wave pattern to facilitate its delineation. However, that strategy may provoke morphological alterations in the P-wave under study that could lead to inaccurate delineation. Alternatively, the present work introduces a new delineation strategy grounded on the generation of a Gaussian model of the Pwave under study to assist its delineation and an adaptive slope threshold that takes into account the morphology of the preceding P-waves. The method was validated using the annotated QT database from Physionet. Delineation results provided a detection sensitivity of 100\%, whereas the mean and standard deviation of the delineation error for the $P$-wave onset, peak and offset were $4.71 \pm 9.59 \mathrm{~ms}$, $2.82 \pm 6.69 \mathrm{~ms}$ and $0.6 \pm 9.79 \mathrm{~ms}$, respectively. These results demonstrate that the proposed strategy provides accurate delineation of $P$-waves that outperforms others presented in the literature, in particular in terms of stability.
\end{abstract}

\section{Introduction}

The P-wave on the electrocardiogram (ECG) represents the electrical activity of the atria and is considered the most reliable non-invasive source of information about atrial conduction [1]. Some morphology characteristics of the Pwave such as its maximum duration or dispersion, among others, have been associated to a higher recurrence and incidence of Atrial Fibrillation (AF), the most common arrhythmia [2]. However, the extraction of information as the boundaries of these waveforms is a complex task due to the absence of standard measurement techniques [2] and the lack of a consensus about the precise definition of the location of these points in the ECG signal. Moreover, manual delineation is a time consuming task with inaccurate results that may vary significantly as a function of the experience and/or fatigue of the physician as well as the presence of noise within the signal [3]. Consequently, this fact has motivated the development of a wide variety of automatic P-wave delineation methods based on different principles. The strategies followed by them range from the use of mathematical tranforms as the phasor transform [4] or the wavelet transform $[3,5]$ to the differentiation of the ECG signal [6], among others.

The method proposed in this study is based on the differentiation of the signal as proposed by Laguna et al. [6], but with decisive differences as the calculation of an adaptive slope threshold that takes into account the morphological characteristics of the preceding P-waves and the creation of a Gaussian model of every P-wave to assists its delineation. In this way, it has been designed an algorithm capable of detecting and delineating accurately a wide variety of P-wave shapes which, in addition, is more respectful with the morphology of the waveforms and more stable than other methods presented in the literature.

\section{Methods}

\subsection{Dataset and preprocessing}

For validation purpose in this study it has been used the standard QT Database (QTDB) [7]. This database contains 105 fifteen-minutes two leads ECG recordings collected from other existing databases with at least 30 manually annotated beats per recording. This database has been chosen as the reference for other P-wave delineator developers for presenting a wide variety of P-wave morphologies and being almost the only free available standard database that contains manual P-wave boundaries annotations. Even though, the lack of accuracy of these annotations, declared as performed at full scope of the two available leads, has been questioned in several previous studies. One recent example can be found in [3]. 
Before the proposed delineation method is applied, the input ECG must be properly conditioned. Initially, the signal is resampled up to $1 \mathrm{kHz}$, the base line is eliminated by subtracting the signal envelope and the power line interference frequency component is removed through adaptive filtering. Later, the high frequency muscle noise is reduced first by applying a wavelet-based method and a bidirectional low-pass filter. The cut-off frequency selected was $70 \mathrm{~Hz}$, a less aggressive filtering than other comparable methods $[3,6]$. This option responds to the demonstrated existence of much higher frequency components in the Pwave than the usually considered [8] and a reduction in the transient effect produced by the proximity of the QRS complex. The last step of the preprocessing is a supervised location of the R-peaks within the signal [9].

\subsection{Delineation algorithm}

In the proposed method, each P-wave is not delineated independently. Instead of that, certain parameters calculated from previously delineated waveforms are used as previous information to guide the location of the fiducial points within a $\mathrm{P}$-wave. Consequently, an initialization step is needed at the beginning of the delineation process in which these parameters are obtained from a reference $\mathrm{P}$-wave, which construction is described next.

\subsection{Reference P-wave construction}

Initially, signal segments prior to the first five R-peaks previously detected are averaged to create a reference segment of the signal. From this, the QRS onset is estimated first and then, prior that point, in a search window of length equal to one third of the median RR distances, the peak with greater amplitude is sought. This point is labelled as the reference P-wave peak. Around that position, a segment of $180 \mathrm{~ms}$ in length is isolated, and will serve as the reference P-wave. Extraordinarily, if the median RR interval is too long $(>900 \mathrm{~ms})$ or too short $(<600 \mathrm{~ms})$ this length is increased or reduced by $20 \mathrm{~ms}$, respectively.

This constructed P-wave might be first categorized as monophasic positive, monophasic negative or biphasic (positive-negative or negative-positive). To do so, a decision algorithm is performed as follows. First, a Gaussian function is generated so that it fits the P-wave in the best way possible. If the fit is good enough, statement that for this method is translated as a Pearson correlation coefficient greater than 0.7 , the waveform is determined to be monophasic positive. Otherwise, the procedure is repeated with the P-wave inverted. In case a proper fit is obtained now, the waveform is classified as monophasic negative. Finally, in case of a new mismatch the waveform is classified as biphasic. For this latter case, the two peaks of the biphasic P-wave are sought forward and backward and the P-wave window is recentered to fit the biphasic morphology. Furthermore, a new Gaussian model to better fit the biphasic wave is created by increasing the order of the Gaussian function. This order is augmented until a Pearson correlation between the P-wave and the Gaussian function higher than 0.7 is reached.

It might be noticed that some P-waves can be asymmetrical. In those cases the Gaussian model will not fit acceptably in any case, which may compromise the delineation performance. When this occur, each half of the Pwave is delineated independently by constructing two artificial waveforms. This is done by meeting both halves with themselves mirrored. Then the delineation of just one half of those artificial waveforms is carried out, as they were distinct P-waves.

To determine the boundaries of the waveform, the Gaussian function is differentiated first and then, the maximum values in each half of the wave are identified, which are the points in which the Gaussian function presents its maximum slopes. Later, based on those values, a slope threshold is calculated and the boundaries of the waveform are determined as the points in which the threshold is exceeded. To determine the mathematical relationship between the maximum slope of a waveform and the slope in its boundaries in each case, a total of $60 \mathrm{P}$-waves were manually delineated by two expert physicians. Thus, this relationship was plotted and the function that resulted to be the best fit, with an R-square score of 0.815 , was simplified to obtain the following equation defining the threshold:

$$
T h(x)=\frac{0.0058 \cdot x}{x+0.012},
$$

where $x$ stands for the waveform maximum slope.

After the Gaussian function has been delineated, the process is repeated with the real $\mathrm{P}$-wave, but restricting the search area for each fiducial point to the vicinity of their position in the model wave. Figure 1 plots an example of this situation in which these intervals are colored in gray. The width of the regions around each fiducial point depends on the goodness of the Gaussian fit. Thus, Figure 1 shows how the area around the offset of the Gaussian model is wider (see offset of this P-wave) as the fit is worse in that half of the wave.

In summary, from the representative P-wave already delineated, the following information is obtained: differences in time and amplitude between the maximum peak of the waveform and its boundaries, the approximated position of the wave with respect to the R-peak, the width of the search window (defined as the width of the waveform, widened a quarter of it on each side), the type of the waveform morphology and some starting coefficients for the Gaussian fit. All this knowledge about the morphology of the P-wave taken as reference will be used to ease the delineation of the P-waves individually. 


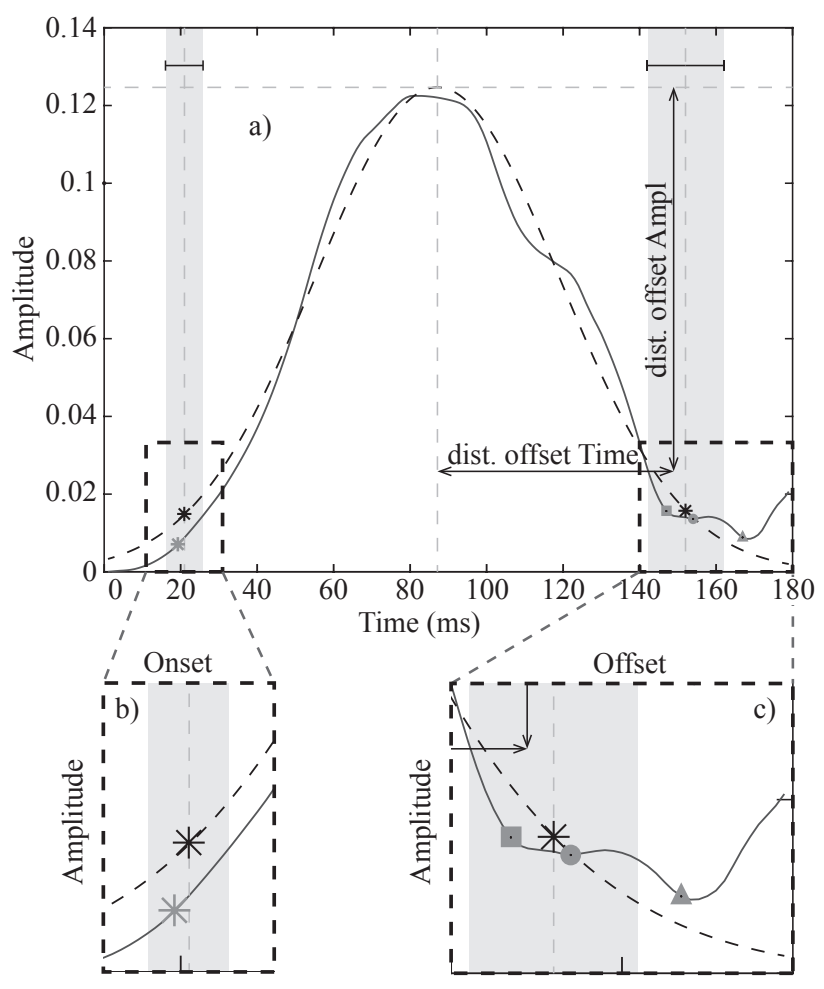

Figure 1. Real P-wave (solid line) and its Gaussian model (dotted line). (a) The detected boundaries on the Gaussian waveform are indicated as black asterisks. The fiducial points search interval is shaded in grey around them. Reference distances in time and amplitude between the peak and the offset computed by taking into account previous waveform morphologies are also displayed. (b) Enlarged beginning with a grey asterisk detected in the real wave as candidate for P-wave onset. (c) Enlarged wave end with different grey shapes are the candidates for P-wave offset.

\subsection{Individualized P-wave delineation}

With all the information obtained from the initialization step, every P-wave is detected in its corresponding search window prior to the R-peak. The delineation method is basically in the same as described before for the reference $\mathrm{P}$-wave. The only difference is the possible existence of more than one fiducial point candidate. In that case, the final decision is based on the morphology parameters that were calculated during the initialization phase. This circumstance is also illustrated in Figure 1.c, where three offset candidates are represented as different grey geometric figures. First, the triangle option would be discarded as it is outside the restricted interval. And then, between the two remaining options, the square option would be selected as it is closer to the point determined by the morphology parameters: distances from peak to offset in time and amplitude obtained from previously delineated P-waves.
Table 1. Comparison of the delineation performance of some of the most relevant $\mathrm{P}$-wave delineation methods in the literature by means of two Validation Parameters (V.P.) making use of the QTDB.

\begin{tabular}{|c|c|c|c|c|}
\hline Methods & V. P. & $\mathrm{P}_{\mathrm{ON}}$ & $\mathrm{P}_{\text {PEAK }}$ & $\mathrm{P}_{\mathrm{OFF}}$ \\
\hline \multirow{2}{*}{\multicolumn{2}{|c|}{ This method $\begin{array}{c}\operatorname{Se}(\%) \\
\mu \pm \sigma(\mathrm{ms})\end{array}$}} & 100 & 100 & 100 \\
\hline & & $4.7 \pm 9.6$ & $2.8 \pm 6.7$ & $0.6 \pm 9.8$ \\
\hline \multirow{2}{*}{$\begin{array}{c}\text { A. Martínez } \\
\text { et al. [4] }\end{array}$} & $\mathrm{Se}(\%)$ & 98.65 & 98.65 & 98.65 \\
\hline & $\mu \pm \sigma(\mathrm{ms})$ & $2.6 \pm 14.5$ & $32 \pm 25.7$ & $0.7 \pm 14.7$ \\
\hline \multirow{2}{*}{$\begin{array}{l}\text { J.P. Martínez } \\
\text { et al. [5] }\end{array}$} & $\mathrm{Z} \quad \mathrm{Se}(\%)$ & 98.87 & 98.87 & 98.87 \\
\hline & $\mu \pm \sigma(\mathrm{ms})$ & $2.0 \pm 14.8$ & $3.6 \pm 13.2$ & $1.9 \pm 12.8$ \\
\hline \multirow{2}{*}{$\begin{array}{l}\text { P. Laguna } \\
\text { et al. [6] }\end{array}$} & $\operatorname{Se}(\%)$ & 97.7 & 97.7 & 97.7 \\
\hline & $\mu \pm \sigma(\mathrm{ms})$ & $14 \pm 13.3$ & $4.8 \pm 10.6$ & $-0.1 \pm 12.3$ \\
\hline
\end{tabular}

Ultimately, once the new P-wave has been delineated, all the parameters obtained which are associated to the reference P-wave are also recomputed with the aim to update some possible variations. An influence ratio of $20 \%$ over the total has been considered. However, before the computations are made, every pair of values are compared. In case the difference is sufficiently large ( $>25 \%$ ), the wave is labeled as abnormal and the refreshing procedure for the reference $\mathrm{P}$-wave is aborted.

\section{Results}

To asses the performance of the proposed algorithm, the delineation error was computed as the difference in time between automatic delineation and manual annotations in the QTDB. For each recording, the larger set of manual annotations in the database was considered. Thus, the global score is presented in terms of the average value of the error $(\mu)$ and its standard deviation $(\sigma)$ as proposed by Martínez et al. [5]. Also the detection performance is evaluated by its Sensitivity ( $\mathrm{Se} \%)$. This parameter indicates the percentage of well detected events. Table 1 shows the results of the proposed method in comparison with other relevant methods presented in the literature.

Results of the proposed method in Table 1 were obtained by using a total of 3176 annotated beats from 96 of the 2-leads ECG recordings from the QTDB. From the original set of 105 recordings, seven of them (sel102, sel221, sel232, sel310, sel36, sel37, sel50) were excluded for the delineation as no P-wave manual annotation were provided and also recordings sello4 and sel36 were not delineated as they did not present a minimum of three consecutive annotated P-waves, a self-imposed condition. 


\section{Discussion}

The delineation results on the QTDB shown in Table 1 have demonstrated that the proposed method is capable of accurately delineate a wide variety of different P-wave morphologies. This has been shown by the more than 3000 waveforms delineated from a large amount of different manually annotated recordings specifically selected to reflect the real world variability. Moreover, it is important to note the excellent results achieved in terms of the standard deviation of the error and sensitivity. It can be observed how the proposed delineation method outperforms the other methods in terms of these two variables. In addition, both for P-wave onset and offset, the obtained standard deviation values are below the acceptable tolerance limits stablished by the CSE working group [10], that are, respectively, 10.2 and $12.7 \mathrm{~ms}$. This exhibits the great stability that brings the use of Gaussian models of the Pwaves as delineation assistants.

On the other hand, with respect to the average value of the error the score obtained, even if still satisfactory, is improved in some cases for those presented by other methods. However, this standard validation parameter might be misleading, since it is likely to benefit from the compensation between earlier and later detections. This risk could be avoided if the mean absolute error was considered instead. Unlike other methods that are based in the use of complex transforms [3-5], the proposed strategy is completely developed in the time domain. This option could be considered more intuitive as it is closer to the way of thinking of physicians when delineating ECGs and, therefore, it could allow the developers to receive feedback more easily for the future improvements of the algorithm.

\section{Conclusions}

In this study, an adaptive $\mathrm{P}$-wave delineation method based on the differentiation of the signal has been presented. The use of information about the historical morphology of the P-waves already delineated and the creation of Gaussian models of every single P-wave to assist its delineation have been revealed as key factors providing higher delineation accuracy and better stability to abnormal P-waves, thus outperforming other methods presented in the literature. Moreover, this algorithm allows a monitoring of the P-wave morphology trend along the ECG and detect anomalous events. Therefore, this method could represent a potencial solution for the identification of progressive changes in the electrical properties of the atria which may help to foresee the occurrence of episodes of arrhythmias, such as atrial fibrillation, or in the clinical decision-making with respect to the diagnosis of cardiovascular diseases related with atrial conduction defects.

\section{Acknowledgements}

Research supported by grants TEC2014-52250-R and DPI2017-83952-C3 MINECO/AEI/FEDER, UE.

\section{References}

[1] Platonov PG. Atrial conduction and atrial fibrillation: What can we learn from surface ECG? Cardiology Journal 2008; 15(5):402-407.

[2] Magnani JW, Williamson MA, Ellinor PT, Monahan KM, Benjamin EJ. P-wave indices: current status and future directions in epidemiology, clinical, and research applications. Circulation Arrhythmia and electrophysiology Feb 2009;2:72-9.

[3] Lenis G, Pilia N, Oesterlein T, Luik A, Schmitt C, Dössel $\mathrm{O}$. P-wave detection and delineation in the ECG based on the phase free stationary wavelet transform and using intracardiac atrial electrograms as reference. Biomedizinische Technik 2016;61(1):37-56.

[4] Martínez A, Alcaraz R, Rieta JJ. Application of the phasor transform for automatic delineation of single-lead ECG fiducial points. Physiological Measurement 2010; 31(11):1467-1485.

[5] Martínez JP, Almeida R, Olmos S, Rocha AP, Laguna P. A wavelet-based ECG delineator evaluation on standard databases. IEEE Transactions on Biomedical Engineering 2004;51(4):570-581.

[6] Laguna P, Jané R, Caminal P. Automatic detection of wave boundaries in multilead ECG signals: Validation with the CSE database. Computers and Biomedical Research 1994; 27(1):45-60.

[7] Laguna P, Mark RG, Goldberg A, Moody GB. Database for evaluation of algorithms for measurement of QT and other waveform intervals in the ECG. In Computers in Cardiology. 1997; 673-676.

[8] Sörnmo L, Laguna P. Chapter 6 - the electrocardiogram-a brief background. In Bioelectrical Signal Processing in Cardiac and Neurological Applications, Biomedical Engineering. Burlington: Academic Press. ISBN 978-0-12-4375529, 2005; $411-452$.

[9] Kohler B, Hennig C, Orglmeister R. The principles of software QRS detection. Engineering in Medicine and Biology Magazine IEEE 2002;21(1):42-57.

[10] The CSE working party. Recommendations for measurement standards in quantitative electrocardiography. European heart journal Oct 1985;6:815-25.

Address for correspondence:

Francisco González Molina

Electronic Engineering Department, Building 7F

Universidad Politécnica de Valencia

Camino de Vera, s/n, 46022, Valencia, Spain

E-mail: fgmolina@upv.es 